Archived version from NCDOCKS Institutional Repository http://libres.uncg.edu/ir/asu/

\title{
Appalachľăn
}

$\overline{\text { B O O N E, NOR T H C A R O L I N A }}$

\section{Flavonol Intake and Cognitive Decline in Middle-Aged Adults}

\author{
Authors: \\ Martin Root, Erin Ravine, and Anne Harper
}

\begin{abstract}
Cognitive decline occurs with age and may be slowed by dietary measures, including increased intake of dietary phytochemicals. However, evidence from large and long-term studies of flavonol intake is limited. Dietary intakes of flavonols were assessed from a large biracial study of 10,041 subjects, aged 45-64, by analysis of a food frequency ques tionnaire administered at visit 1 of triennial visits. Cognitive function was assessed at visits 2 and 4 with the following three cognitive performance tests: the delayed word recall test, the revised Wechsler Adult Intelligence Scale digit symbol subtest, and the word fluency test of the Multilingual Aphasia Examination. The change in each score over 6 years was calculated, and a combined standardized change score was calculated. Generalized linear models controlled for age, ethnicity, gender, education level, energy intake, current smoking, physical activity,

body mass index, diabetes, and vitamin $\mathrm{C}$ intake. Total flavonols across quintiles of intake were positively associated with preserved combined cognitive function $(\mathrm{P}<.001)$. This pattern with preserved combined cognitive function was consistent for the three major individual flavonols in the diet, myricetin, kaempferol, and quercetin (each $\mathrm{P}<.001$ ). The positive association with total flavonols was strongest for the digit symbol subtest $(\mathrm{P}<.001)$. In this cohort, flavonol intake was correlated with protected cognitive function over time
\end{abstract}

Martin Root, Erin Ravine, and Anne Harper(2015) Flavonol Intake and Cognitive Decline in Middle-Aged Adults. JOURNAL OF MEDICINAL FOOD [DOI 10.1089] copy available @ (http:// online.liebertpub.com/doi/pdfplus/10.1089/jmf.2015.0010) 


\title{
Flavonol Intake and Cognitive Decline in Middle-Aged Adults
}

\author{
Martin Root, Erin Ravine, and Anne Harper
}

\begin{abstract}
Cognitive decline occurs with age and may be slowed by dietary measures, including increased intake of dietary phytochemicals. However, evidence from large and long-term studies of flavonol intake is limited. Dietary intakes of flavonols were assessed from a large biracial study of 10,041 subjects, aged 45 - 64, by analysis of a food frequency ques tionnaire administered at visit 1 of triennial visits. Cognitive function was assessed at visits 2 and 4 with the following three cognitive performance tests: the delayed word recall test, the revised Wechsler Adult Intelligence Scale digit symbol subtest, and the word fluency test of the Multilingual Aphasia Examination. The change in each score over 6 years was calculated, and a combined standardized change score was calculated. Generalized linear models controlled for age, ethnicity, gender, education level, energy intake, current smoking, physical activity, body mass index, diabetes, and vitamin $\mathrm{C}$ intake. Total flavonols across quintiles of intake were positively associated with preserved combined cognitive function $(P<.001)$. This pattern with preserved combined cognitive function was consistent for the three major individual flavonols in the diet, myricetin, kaempferol, and quercetin (each $P<.001$ ). The positive association with total flavonols was strongest for the digit symbol subtest $(P<.001)$. In this cohort, flavonol intake was correlated with protected cognitive function over time.
\end{abstract}

\section{INTRODUCTION}

$\mathrm{C}$ ognitive decline is highly associated with the aging process and is a common health concern among older adults. Although its rate and degree of severity vary among individuals, its presence is seen in decreased neurocognitive performance, increased neurodegenerative diseases, physical disabilities, a loss of independence, and a decreased quality of life.

There are many risk factors for cognitive decline. In ad dition to the nonmodifiable risk factors of age and genetic

predisposition, factors such as hypertension, smoking, and diabetes have been associated with increased rates of cog nitive decline. ${ }^{3,4}$ Higher education level, literacy level, and physical activity have been shown to be associated with a slower rate of cognitive decline. ${ }^{5,6}$ These factors may be linked through the interrelationship between vascular function and neuroinflammation.

Certain dietary risk factors have been linked to cognitive function. Ortega et al. showed that among the elderly, better cognitive scores were associated with greater intakes of total food, carbohydrate, fiber, folate, vitamin $\mathrm{C}, \mathrm{b}$-carotene, iron, and zinc and with lower intakes of saturated fatty acids. ${ }^{9}$ Similarly, Scarmeas et al. found that high adherence to the
Mediterranean diet, a diet high in olive oil, legumes, unre fined cereals, fruits, vegetables, and fish, led to a $28 \%$ lower risk of developing mild cognitive impairment and a $48 \%$ lower risk for developing Alzheimer' s disease compared to subjects with low adherence. ${ }^{10}$

These dietary factors may be acting through oxidative stress and neuroinflammation, resulting in cognitive decline and neurodegenerative diseases. Consumption of antioxidants may help to protect againsst oxidative stress in the brain, slowing cognitive decline. Vitamins E, C, and bcarotene have been studied for their relation to cognitive function with mixed results. A cross-sectional study by Peacock et al. used the Atherosclerosis Risk in Communities (ARIC) study to examine antioxidant intake, supplement use, and cognitive function. They found that the intake of carotenoids and vitamins $\mathrm{C}$ and $\mathrm{E}$ had no effect on cognitive performance. positive association between the plasma levels of these an tioxidants and the cognitive performance tests and con cluded that the serum levels of these antioxidants were significant predictors of memory performance. These results were not associated with supplemental intake because of the small $(6 \%)$ number of their population that mentioned supplement use..$^{15}$ Since these levels of plasma antioxidants may have represented an intake of fruits and vegetables, it is plausible that memory performance in these populations was related to the intake of fruits and vegetables in general. The fruits and vegetables also contain flavonols and other polyphenols. 
Flavonols have antioxidant properties; these are plantbased polyphenolic compounds that can be found in foods and beverages such as blueberries, tea, wine, and fruit jui ces. Passing through the blood - brain barrier, flavonols and their metabolites can have positive effects on cell-to-cell communication, vascular function, and neuroinflammation, through the inhibition of specific enzymes and the activation of specific signaling pathways in the brain. These mecha nisms have been proposed to increase neuronal integrity, ultimately deterring brain aging and cognitive decline. ${ }^{16}$

Research has been conducted on the effects of flavonoid consumption on neurocognitive performance, cognitive decline, and prevention of neurodegenerative diseases. Previous animal studies found that flavonoid consumption was associated with increased neurocognitive performance and decreased incidences of age-related cognitive decline. ${ }^{16,17}$ In addition, the PAQUID study found high intakes of fla vonoids to be associated with slowed incidences of cognitive decline over a 10-year period in a population of 1640 older adults $(P=.046){ }^{18}$

Our research will further investigate these findings in a biracial longitudinal study of over 10,000 middle-aged adults. Our hypothesis is that high intake of dietary flavonols in middle-aged adults is associated with decreased rates of cognitive decline over time.

\section{MATERIALS AND METHODS}

\section{Participants}

This article was prepared using ARIC Research Materials obtained from the National Heart Lung Blood Institute (NHLBI) Biologic Specimen and the Data Repository Information Coordinating Center and does not necessarily reflect the opinions or views of the ARIC research groups or the NHLBI. The Institutional Review Board of Appalachian State University approved the acquisition and use of this dataset. Details of the methodology of the ARIC study are described elsewhere. ${ }^{19}$

The ARIC study is a prospective epidemiological study that explores the causes and outcomes of atherosclerosis in four communities located in the United States. These are Washington County, Maryland, Forsyth County, North Carolina, the city of Jackson, Mississippi, and selected northwestern suburbs of Minneapolis, Minnesota. The study protocol was approved by institutional review boards at each clinical site.

Study participants underwent extensive examinations, including measurements of cardiovascular conditions, var ious risk factors, a dietary questionnaire, and cognitive function tests. Baseline examinations occurred from 1987 to 1989 at visit 1, and reexaminations took place from 1990 to 1992 for visit 2, 1993 to 1995 for visit 3, and 1996 to 1998 for visit 4. Specific ARIC data that are of particular interest to this study include dietary intake and certain covariates from visit 1 and data on cognitive function from visits 2 to 4 . A food frequency questionnaire (FFQ) was not administered at visit 2 .

\section{Dietary intake}

Dietary assessment was conducted by trained interviewers with use of a 66-item FFQ during visit 1. Nutrient analysis of the FFQ was completed with use of Willett' $s$ nutritional database. The analysis provided information on 90 different nutrients, including the nutrients of interest for this study, which were the major flavonols myricetin, kaempferol, and quercetin. Flavonol intakes were calculated by multiplying the individual flavonol content of each food item by the frequency of its daily consumption and summing over all items.

\section{Cognition}

Measures of cognitive function were assessed with the use of three cognitive performance tests: the delayed word recall test, the revised Wechsler Adult Intelligence Scale (WAIS-R) digit symbol subtest, and the word fluency test of the Multilingual Aphasia Examination. ${ }^{22}$ The tests were administered by trained interviewers in a standardized order in a quiet room. Interviewer performance was monitored by tape recording, reviewing a sample of the testing sessions, and by confirming that there were no systematic differences in mean scores obtained by the different interviewers who gave the tests. ${ }^{23}$

The delayed word recall test is a test of verbal learning and recent memory that requires the participant to recall 10 common nouns following a 5-min interval during which another psychometric test is given. After the $5 \mathrm{~min}$ and to standardize the elaborative processing of the words to be recalled, individuals are asked to compose sentences incorporating the nouns as they were presented. A point is given for each word recalled, with a maximum of 10 points. This test has been shown to have a high 6-month test - retest reliability in a study, including 26 normal elderly persons $(R=0.75) .^{24}$

The WAIS-R digit symbol subtest is a paper and pencil task requiring timed translation of numbers 1 - 9 to symbols using a key. The test measures psychomotor performance and is relatively unaffected by intellectual ability, memory, or learning for most adults. ${ }^{25}$ It is scored as the number of numbers translated to symbols correctly within $90 \mathrm{sec}$, with a maximum score of 93. Short-term test - retest reliability has been found to be high in middle-aged individuals $(R=0.82){ }^{25}$

The word fluency test requires participants to generate as many words as possible in $60 \mathrm{sec}$, beginning with a letter from the alphabet. Three trials using the letters F, A, and $\mathrm{S}$ were conducted, and word fluency was scored as the total number of words generated over the three trials. The test is sensitive to linguistic impairment ${ }^{26}$ and early mental decline in older persons. ${ }^{27}$ The immediate test - retest correlation coefficient based on an alternate test form has been found to be high $(R=0.82){ }^{28}$

For the present study, the three test scores were stan dardized by taking the difference of the test scores at visit 2 from visit 4 and dividing by the standard deviation of the difference. A summed score of change in cognitive function 
was created by summing the three standardized scores for each subject.

\section{Other measurements}

Education level, ethnicity, and presence of diabetes and hypertension were assessed by standardized interviews during baseline examinations. Education levels were categorized into the following three groups: $£ 11$ years of education, $12-16$ years of education, and $\ddagger 17$ years of education. Presence of diabetes was defined as serum fasting glucose $\ddagger 126 \mathrm{mg} / \mathrm{dL}$, self-reported physician diagnosis of diabetes, or use of oral hypoglycemic agents. Hypertension was defined as systolic blood pressure $\ddagger 140 \mathrm{mmHg}$, dia stolic blood pressure $\$ 90 \mathrm{mmHg}$, or in a person taking antihypertensive medication. Black ethnicity was self-reported and defined as black or nonblack. Current smoking was selfreported. Prevalent coronary heart disease was identified by a complex algorithm involving electrocardiogram re sults and self-reported heart conditions. Body mass index (BMI) was calculated as $\mathrm{kg} / \mathrm{m}^{2}$. Total calories and vitamin C intake were assessed from the FFQ. Physical activity was assessed as the sum of work-related, leisure, and sports activity scores.

\section{Statistical analysis}

A total of 15,750 individuals between the ages of 45 and 64 years at baseline were available for this analysis. A large number of subjects were lost to the analysis if cognitive function scores were unavailable, principally from the fourth visit (4755). Subjects were lost to the analysis for missing data from the FFQ (216), physical activity (39), diabetes diagnoses (73), smoking status (9), and educational status (15). Also, excluded were those whose change scores in cognitive function were greater than or less than five standard deviations from zero (33). These were removed for a concern over reverse causality and an abundance of concern about excessive influence in the models.

With use of the statistical software SPSS version 20.0, generalized linear models of change in cognitive function versus flavonol intake were performed. Controlling factors included age, ethnicity, gender, educational level, energy intake, current smoking, physical activity, BMI, diabetes di agnosis, and vitamin $\mathrm{C}$ intake. The key outcomes in Tables 3 and 4 were expressed as relative changes in the standardized cognitive change scores compared to group 5, the highest intake group of flavonols. A model adjusting for only gender and age was prepared, but showed little difference from the unadjusted model; therefore, the results were not presented.

\section{RESULTS}

A total of 10,041 subjects were included in the analysis after exclusions. As seen in Table 1, there were significant trends in subject characteristics across quintiles of total flavonol intake. As flavonol intake increased, the percentage of black ethnicity and smokers decreased and the percentage of males, level of education, total calories, age, and physical activity increased.

Cognitive function scores were consistently lower for women compared to men after controlling for important confounders (Table 2). Women' s scores decreased more rapidly than men' $\mathrm{s}$ for words recalled and word fluency,

Table 1. Characteristics of ARIC Cohort by Quintiles of Total Flavonol Intake

\begin{tabular}{|c|c|c|c|c|c|c|}
\hline \multirow[b]{2}{*}{ Variable } & \multicolumn{5}{|c|}{ Quintiles of dietary intake of total flavonols } & \multirow[b]{3}{*}{ P-value for trend* } \\
\hline & 1 & 2 & 3 & 4 & 5 & \\
\hline$N$ & 2008 & 2006 & 2004 & 2008 & 2015 & \\
\hline Total flavonols mean intake, $\mathrm{mg} /$ day & 2.8 & 6.2 & 9.7 & 15.1 & 34.7 & \\
\hline \multicolumn{7}{|l|}{ Percentages } \\
\hline Black ethnicity & 26.0 & 19.0 & 18.6 & 16.8 & 12.5 & $<.001$ \\
\hline Male gender & 50.0 & 47.9 & 42.7 & 42.8 & 40.5 & $<.001$ \\
\hline Current smokers & 28.4 & 20.6 & 18.7 & 19.5 & 19.5 & $<.001$ \\
\hline Diabetes & 8.5 & 8.9 & 9.5 & 8.1 & 9.0 & .065 \\
\hline Education $>16$ years & 35.1 & 38.8 & 41.7 & 41.9 & 39.4 & $<.001$ \\
\hline \multicolumn{7}{|l|}{ Means } \\
\hline Age, visit 1 & 53.5 & 54.0 & 54.2 & 54.2 & 54.0 & $<.001$ \\
\hline BMI, $\mathrm{kg} / \mathrm{m}^{2}$ & 27.5 & 27.3 & 27.3 & 27.6 & 27.6 & .018 \\
\hline Total energy intake, $\mathrm{kJ} /$ day & 345 & 375 & 396 & 397 & 434 & $<.001$ \\
\hline Physical Activity sum of activity codes & 6.90 & 7.11 & 7.19 & 7.22 & 7.13 & $<.001$ \\
\hline \multirow[t]{2}{*}{ Vitamin $\mathrm{C}$ intake, $\mathrm{mg} /$ day } & 78 & 109 & 133 & 134 & 155 & $<.001$ \\
\hline & \multicolumn{5}{|c|}{ Quintiles of dietary intake of flavonols (mean intake, $\mathrm{mg} /$ day) } & \\
\hline Myrictin & 0.05 & 0.27 & 0.52 & 1.03 & 2.69 & \\
\hline Kaempferol & 0.2 & 0.8 & 2.1 & 4.1 & 11.6 & \\
\hline Quercetin & 1.9 & 4.2 & 6.7 & 9.7 & 20.9 & \\
\hline
\end{tabular}

*Controlled for age, ethnicity, gender, education level, energy intake, and current smoking where appropriate.

ARIC, Atherosclerosis Risk in Communities; BMI, body mass index. 
Table 2. Measures of Cognitive Function and Changes in Cognitive Function Over 6 Years Comparing Men and Women in the ARIC Cohort

\begin{tabular}{lccc}
\hline Cognitive measure & Men & Women & $\begin{array}{c}\text { P for } \\
\text { difference* }\end{array}$ \\
\hline Visit 2 & & & \\
$\quad$ Words recalled & $6.97-1.42$ & $6.42-1.46$ & $<.001$ \\
$\quad$ Digit symbols & $48.2-13.7$ & $43.9-12.5$ & $<.001$ \\
$\quad$ Words listed & $34.7-12.0$ & $33.5-12.3$ & $<.001$ \\
Visit 4 & & & \\
$\quad$ Words recalled & $6.86-1.54$ & $6.22-1.57$ & $<.001$ \\
Digit symbols & $45.5-13.6$ & $41.4-12.4$ & $<.001$ \\
$\quad$ Words listed & $34.4-12.1$ & $32.6-12.7$ & $<.001$ \\
Changes & & & \\
$\quad$ Words recalled & $-0.12-1.55$ & $-0.20-1.54$ & .033 \\
Digit symbols & $-2.65-6.89$ & $-2.51-6.30$ & .017 \\
$\quad$ Word fluency & $-0.25-7.78$ & $-0.89-7.83$ & $<.001$ \\
Global total change score $-0.47-1.75$ & $-0.58-1.70$ & .043 \\
\hline
\end{tabular}

*Controlled for age, ethnicity, education level, energy intake, and current smoking.

while men' s scores in digit symbols decreased more rapidly than for women. The overall standardized change score was slightly lower for women than men.

Standardized total cognitive change scores were lower (more rapid decline in cognitive function) in lower quintiles of total flavonol intake when controlling for a number of important covariates (Table 3). The results were consistent for the three major individual flavonols.

Across quintiles of total flavonols, the individual cognitive function change scores gave variable results with changes in digit symbols being most significantly correlated with flavonol intake as seen in Table 4.

\section{DISCUSSION}

In this population of middle-aged adults, preserved cog nitive function was significantly associated with increased

Table 3. Standardized Cognitive Change Score Over 6 Years in Quintiles of Intake of Flavonols in the ARIC Cohort

\begin{tabular}{|c|c|c|c|c|c|c|}
\hline & \multicolumn{5}{|c|}{ Quintiles of flavonol intake } & \multirow{2}{*}{$\begin{array}{l}\mathrm{P} \text { for } \\
\text { trend }\end{array}$} \\
\hline & 1 & 2 & 3 & 4 & 5 & \\
\hline Total flavonols & $\begin{array}{c}-0.173^{*} \\
-0.211^{*}\end{array}$ & $\begin{array}{c}-0.184 * \\
-0.191 *\end{array}$ & $\begin{array}{l}-0.220^{*} \\
-0.214^{*}\end{array}$ & $\begin{array}{l}-0.032 \\
-0.028\end{array}$ & $\begin{array}{l}0 \\
0\end{array}$ & $\begin{array}{l}<.001 \\
<.001\end{array}$ \\
\hline Myricetin & $\begin{array}{l}-0.244^{*} \\
-0.266^{*}\end{array}$ & $\begin{array}{l}-0.232 * \\
-0.238 *\end{array}$ & $\begin{array}{l}-0.121 * \\
-0.118 *\end{array}$ & $\begin{array}{r}0.009 \\
-0.004\end{array}$ & $\begin{array}{l}0 \\
0\end{array}$ & $\begin{array}{l}<.001 \\
<.001\end{array}$ \\
\hline Kaempferol & $\begin{array}{l}-0.240^{*} \\
-0.246^{*}\end{array}$ & $\begin{array}{l}-0.187 * \\
-0.172 *\end{array}$ & $\begin{array}{l}-0.323^{*} \\
-0.304 *\end{array}$ & $\begin{array}{l}-0.084 \\
-0.068\end{array}$ & $\begin{array}{l}0 \\
0\end{array}$ & $\begin{array}{l}<.001 \\
<.001\end{array}$ \\
\hline Querc & $\begin{array}{l}-0.104 \\
-0.145^{*}\end{array}$ & $\begin{array}{l}-0.210^{*} \\
-0.225^{*}\end{array}$ & $\begin{array}{l}-0.150 * \\
-0.151 *\end{array}$ & $\begin{array}{l}-0.055 \\
-0.049\end{array}$ & $\begin{array}{l}0 \\
0\end{array}$ & $\begin{array}{l}.001 \\
.001\end{array}$ \\
\hline
\end{tabular}

Analysis was performed using generalized linear models. The first row in each group was an unadjusted model. The second row was adjusted for age, ethnicity, gender, education level, energy intake, current smoking, physical activities, BMI, diabetes, and vitamin $\mathrm{C}$ intake. Asterisks (*) indicate a significant difference with quintile 5 at $P<.05$.
Table 4. Changes in Cognitive Functions Over 6 Years in Quintiles of Total Flavonol Intake in the ARIC Cohort

\begin{tabular}{|c|c|c|c|c|c|}
\hline \multirow[b]{2}{*}{ Cognitive function } & \multicolumn{4}{|c|}{ Quintiles of total flavonol intake } & \multirow{2}{*}{$\overline{5} \begin{array}{l}\mathrm{P} \text { for } \\
\text { trend }^{*}\end{array}$} \\
\hline & 1 & 2 & 3 & 4 & \\
\hline $\begin{array}{l}\text { Total cognitive } \\
\text { function }\end{array}$ & $\begin{array}{l}-0.173 * \\
-0.211 *\end{array}$ & $\begin{array}{l}-0.184 * \\
-0.191 *\end{array}$ & $\begin{array}{l}-0.220 * \\
-0.214 *\end{array}$ & $\begin{array}{l}-0.0320 \\
-0.0280\end{array}$ & $\begin{array}{l}<.001 \\
<.001\end{array}$ \\
\hline Words recalled & $\begin{array}{l}-0.008 \\
-0.012\end{array}$ & $\begin{array}{r}-0.009 \\
0.009\end{array}$ & $\begin{array}{l}-0.029 \\
-0.027\end{array}$ & $\begin{array}{c}0.0150 \\
0.0180\end{array}$ & $\begin{array}{l}.569 \\
.582\end{array}$ \\
\hline Digitsy & $\begin{array}{l}-0.107 * \\
-0.167 *\end{array}$ & $\begin{array}{l}-0.088 * \\
-0.115 *\end{array}$ & $\begin{array}{l}-0.121 * \\
-0.133 *\end{array}$ & $\begin{array}{l}-0.0310 \\
-0.0400\end{array}$ & $\begin{array}{l}<.001 \\
<.001\end{array}$ \\
\hline Word fluency & $\begin{array}{l}-0.058 \\
-0.033\end{array}$ & $\begin{array}{l}-0.087 * \\
-0.067 *\end{array}$ & $\begin{array}{l}-0.070 * \\
-0.054\end{array}$ & $\begin{array}{l}-0.0170 \\
-0.0060\end{array}$ & $\begin{array}{r}.027 \\
.137\end{array}$ \\
\hline
\end{tabular}

Analysis was performed using generalized linear models. The first row in each group was an unadjusted model. The second rows were adjusted for age, ethnicity, gender, education level, energy intake, current smoking, physical activities, BMI, diabetes, and vitamin $\mathrm{C}$ intake. Asterisks $\left(^{*}\right)$ indicate a significant difference with quintile 5 at $P<.05$.

flavonol intake. In particular, standardized total cognitive decline did not decline as fast with increased intake of total flavonols and the individual intakes of myricetin, kaempferol, and quercetin. Our findings support evidence that greater intake of flavonol-containing foods may reduce cognitive decline in the middle aged and supports the rela tionship of specific phytochemicals with cognition. ${ }^{29}$ Previous studies have been limited by their short length of follow-up, small sample sizes, elderly populations, and minimal adjustments for confounding factors. In the pres ent study, we compared a standardized change score over 6 years with initial dietary flavonol intake in a large, biracial middle-aged population. Although causation cannot be at tributed, it is interesting to note that the adjusted difference in global cognitive change between the first and fifth quintile for total flavonols $(-0.211)$ represents about 2.4 years of the preserved change in standardized cognitive function seen in the cohort over 6 years $(-0.52)$.

The trajectory of cognitive function was measured by a combined change score for the delayed word recall test, the WAIS-R digit symbol subtest, and the word fluency test of the Multilingual Aphasia Examination. Advantages of using a combined score of change in cognitive function are comprehensiveness and stability, since many overlapping areas of brain function contribute to these cognitive abilities and their change over time. Similar combined scores have been calculated and used in other studies for analysis of cognitive function. ${ }^{30,31}$ The change in the test score for the digit symbol was positively associated with increased total flavonol intake $(P$-values $<.001)$. Change in word fluency was only marginally associated with flavonol intake $(P=$ .27 for the unadjusted model and not significantly corre lated for the adjusted models). Changes in word recall were not associated with flavonol intake.

Previous studies investigating the relationship between cognition and plant compounds, including antioxidants, B vitamins, vitamin D, fatty acids, and other phytochemicals, 
have yielded mixed results. ${ }^{32,33}$ Generally, a trend is seen toward the benefits of plant consumption in a wellbalanced diet. The present results support previous research related to fruit and vegetable compounds and cognition, although specific to flavonols in a large diverse population. In the ARIC, cohort flavonols were most commonly consumed in fruits and vegetables, particularly apples and broccoli. Tea was an especially rich source.

Women experienced slightly greater overall cognitive decline over the 6-year study $(P=.043)$, despite their greater total flavonol intake. This gender difference was driven largely by the differences in delayed word fluency test de cline, while the cognitive decline correlation with flavonol intake was driven largely by the change in digit symbols. Despite these gender differences, both genders had more preserved overall cognitive function with higher total fla vonol intake.

Foods, particularly fruits and vegetables, contain a num ber of compounds with putative salutary effects, including acting as an antioxidant. The flavonols in question in this study have measurable antioxidant activity. ${ }^{16,18}$ However, previous work with the ARIC cohort showed that dietary antioxidants did not protect against cognitive decline. ${ }^{14}$ However, other work has shown that flavonoids have a wide range of other possible neuroprotective effects. These pri marily work by reducing neuroinflammation and associated cytokine release. In addition, neural signaling pathways have been shown to be activated together with neurogenesis and improved blood flow through inducible nitric oxide synthase activation. ${ }^{16,34}$ Vitamin $\mathrm{C}$ was included as a co factor in the analysis as the major water-soluble antioxidant nutrient in these fruits and vegetables. By controlling for this antioxidant, this analysis was able to show that the corre lations seen were independent of this major antioxidant in the food sources of flavonols.

The current study was limited by having only one test for each cognitive domain. Within the tests that were taken, there is the potential for a lack of correspondence between the cognitive scores and the latent cognitive abilities they were meant to measure. ${ }^{35}$ Some of these limitations are corrected for by the combined, standardized, and total cognitive decline score. The dietary assessment also pre ceded the first cognitive measure by 3 years. Although the statistical model adjusts for a number of variables, it cannot control for all possible known and unknown confounding variables that may contribute to changes in cognition. Fi nally, this is a strictly observational study and cause and effect cannot be implied from this analysis.

Our data support existing research on the consumption of fruits and vegetables, and also possibly tea, and cognitive decline. An overall increased consumption of flavonols was significantly correlated with slower cognitive decline in this large, diverse middle-aged population.

\section{AUTHOR DISCLOSURE STATEMENT}

This research received no specific grant from any funding agency, commercial sectors, or not-for-profit sectors.

\section{REFERENCES}

1. Singh-Manoux A, Kivimaki M, Glymour MM, et al:: Timing of onset of cognitive decline: Results from Whitehall II prospective cohort study. BMJ 2012;344:d7622.

2. Bishop NA, Lu T, Yankner BA: Neural mechanisms of ageing and cognitive decline. Nature 2010;464:529 - 535.

3. Cherbuin N, Reglade-Meslin C, Kumar R, et al:: Risk factors of transition from normal cognition to mild cognitive disorder: The PATH through Life Study. Dement Geriatr Cogn Disord 2009; 28:47 - 55.

4. Gregg EW, Yaffe K, Cauley JA, et al.: Is diabetes associated with cognitive impairment and cognitive decline among older women? Study of Osteoporotic Fractures Research Group. Arch Intern Med 2000;160:174 - 180.

5. Yaffe K, Fiocco AJ, Lindquist K, et al:: Predictors of maintaining cognitive function in older adults: The Health $\mathrm{ABC}$ study. Neurology 2009;72:2029 - 2035.

6. Sofi F, Valecchi D, Bacci D, et al:: Physical activity and risk of cognitive decline: A meta-analysis of prospective studies. $J$ Intern Med 2011;269:107 - 117.

7. Yaffe K, Kanaya A, Lindquist K, et al:: The metabolic syndrome, inflammation, and risk of cognitive decline. JAMA 2004;292: $2237-2242$.

8. Ownby R: Neuroinflammation and cognitive aging. Curr Psychiatry Rep 2010;12:39 - 45.

9. Ortega RM, Requejo AM, Andres P, et al:: Dietary intake and cognitive function in a group of elderly people. Am J Clin Nutr 1997;66:803-809.

10. Scarmeas N, Stern Y, Mayeux R, et al.: Mediterranean diet and mild cognitive impairment. Arch Neurol 2009;66:216 - 225.

11. Mangialasche F, Polidori MC, Monastero R, et al.: Biomarkers of oxidative and nitrosative damage in Alzheimer' s disease and mild cognitive impairment. Ageing Res Rev 2009;8:285 - 305.

12. Head E: Oxidative damage and cognitive dysfunction: Antioxidant treatments to promote healthy brain aging. Neurochem Res 2009;34:670 - 678.

13. Farooqui T, Farooqui AA: Aging: An important factor for the pathogenesis of neurodegenerative diseases. Mech Ageing Dev 2009; 130:203 - 215.

14. Peacock JM, Folsom AR, Knopman DS, et al.: Dietary antioxidant intake and cognitive performance in middle-aged adults. The Atherosclerosis Risk in Communities (ARIC) Study investigators. Publ Health Nutr 2000;3:337 - 343.

15. Perrig WJ, Perrig P, Stahelin HB: The relation between antioxidants and memory performance in the old and very old. $J$ Am Geriatr Soc 1997;45:718 - 724.

16. Williams RJ, Spencer JP: Flavonoids, cognition, and dementia: Actions, mechanisms, and potential therapeutic utility for Alzheimer disease. Free Radic Biol Med 2012;52:35 - 45.

17. Camfield DA, Scholey A, Pipingas A, et al:: Steady state visually evoked potential (SSVEP) topography changes associated with cocoa flavanol consumption. Physiol Behav 2012;105:948 - 957.

18. Letenneur L, Proust-Lima C, Le Gouge A, et al: Flavonoid intake and cognitive decline over a 10-year period. Am J Epidemiol 2007; $165: 1364$ - 1371.

19. The ARIC Investigators: The Atherosclerosis Risk in Communities (ARIC) Study: Design and objectives. Am J Epidemiol 1989;129:687 - 702.

20. ARIC: ARIC Dietary Intake Form. 1986. www2.cscc.unc.edu/aric/ sites/default/fi s/public/forms/DTIA.pdf (accessed August 12, 2014). 
21. ARIC: ARIC Data Book: Cohort, Exam 1 Nutrition Derived Variables in ANUT2. 2010. www2.cscc.unc.edu/aric/sites/default/ files/public/datasets/ANUT2.pdf (accessed August 14, 2014).

22. Benton A: Multilingual Aphasia Examination. 2nd ed. AJA Associates, Iowa City, 1989.

23. ARIC: ARIC Cognitive Function Form. 2010. www2.cscc.unc .edu/aric/sites/default/files/public/datasets/CNFA.pdf (accessed August 12, 2014).

24. Knopman DS, Ryberg S: A verbal memory test with high predictive accuracy for dementia of the Alzheimer type. Arch Neurol 1989;46:141 - 145.

25. Wechsler D: WAISR Manual. The Psychological Corporation, Cleveland, OH, 1981.

26. Tranel D: Neuropsychological assessment. Psychiatr Clin North Am 1992;15:283 - 299.

27. Benton AL, Eslinger PJ, Damasio AR: Normative observations on neuropsychological test performances in old age. J Clin Neuropsychol 1981;3:33- 42.

28. Franzen M: Multilingual aphasia examination. In: Test Critiques (Keyser D and Sweetland R, eds.). Test Corporation of America, Kansas City, MO, 1986, pp. 278 - 282.
29. Crichton GE, Bryan J, Murphy KJ: Dietary antioxidants, cognitive function and dementia-A systematic review. Plant Foods Hum Nutr 2013;68:279 - 292.

30. Naorungroj S, Slade GD, Beck JD, et al:: Cognitive decline and oral health in middle-aged adults in the ARIC study. J Dent Res 2013;92:795 - 801.

31. Hosking DE, Nettelbeck T, Wilson C, et al:: Retrospective lifetime dietary patterns predict cognitive performance in communitydwelling older Australians. Br J Nutr 2014;112:228 - 237.

32. Devore EE, Kang JH, Stampfer MJ, et al:: The association of antioxidants and cognition in the Nurses' Health Study. Am J Epidemiol 2013;177:33-41.

33. Gillette-Guyonnet S, Secher M, Vellas B: Nutrition and neurodegeneration: Epidemiological evidence and challenges for future research. Br J Clin Pharmacol 2013;75:738 - 755.

34. Spencer JP, Vafeiadou K, Williams RJ, et al:: Neuroinflammation: Modulation by flavonoids and mechanisms of action. Mol Aspects Med 2012;33:83 - 97.

35. Proust-Lima C, Dartigues JF, Jacqmin-Gadda H: Misuse of the linear mixed model when evaluating risk factors of cognitive decline. Am J Epidemiol 2011;174:1077 - 1088. 